\title{
Clinical significance of chromodomain helicase/ATPase DNA binding protein 1-like and human mutL homolog 1 gene expression in cholangiocarcinoma
}

\author{
JINGWEN HUA ${ }^{1}$, SHINIAO LI $^{2}$ and CHANGWEN HUANG ${ }^{2}$ \\ ${ }^{1}$ Department of Oncological Surgery, Xinyi People's Hospital, Xuzhou, Jiangsu 221400; \\ ${ }^{2}$ Department of Hepatobiliary Surgery, Jiangxi Provincial People's Hospital, Nanchang, Jiangxi 330006, P.R. China
}

Received December 8, 2015; Accepted June 6, 2018

DOI: $10.3892 / \mathrm{ol} .2018 .9043$

\begin{abstract}
Cholangiocarcinoma is a highly malignant form of gastrointestinal cancer with an unfavorable prognosis. The novel oncogene chromodomain helicase/ATPase DNA binding protein 1-like (CHD1L) has been confirmed to serve a vital role in numerous types of cancer, including liver cancer. Mismatch repair (MMR) is a common DNA repair process that contributes to the preservation of the integrity and stability of genetic substances. Human mutL homolog 1 gene (hMLH1) is an important MMR protein family member. The present study aimed to evaluate the pathological and clinical features of cholangiocarcinoma, and to investigate the clinical significance of CHD1L and hMLH1 expression in cholangiocarcinoma. A total of 108 samples from cholangiocarcinoma tumor tissues and 60 samples from normal bile duct tissue were obtained from patients admitted to The Second Affiliated Hospital of Nanchang University between May 2005 and May 2014. All cholangiocarcinoma cases were pathologically confirmed. The expression of CHD1L and hMLH1 was examined by immunohistochemistry analysis. The expression of CHD1L in cholangiocarcinoma (94.44\%) was significantly higher than in normal bile duct tissues (40.00\%). CHD1L expression was associated with gallstone history, serum carbohydrate antigen 19-9 (CA19-9) level and Tumor-Node-Metastasis $(\mathrm{TNM})$ stage $(\mathrm{P}<0.05)$. hMLH1 expression in cholangiocarcinoma $(77.78 \%)$ was significantly lower than in normal bile duct tissues (96.67\%), and was associated with gender, age, serum CA19-9 level, the presence of hepatitis B virus surface antigen, TNM stage and tumor diameter $(\mathrm{P}<0.05)$. Kaplan-Meier survival curve analysis
\end{abstract}

Correspondence to: Dr Changwen Huang, Department of Hepatobiliary Surgery, Jiangxi Provincial People's Hospital, 92 Ai Guo Road, Nanchang, Jiangxi 330006, P.R. China

E-mail: ncdxhcw@163.com

Key words: cholangiocarcinoma, clinical significance, prognosis, CHD1L, hMLH1 indicated that the 3-year accumulative survival rates for CHD1L-positive and -negative patients differed significantly $(\mathrm{P}<0.05 ; 17.90$ and $83.33 \%$, respectively). There was no statistically significant difference $(\mathrm{P}>0.05)$ between the 3 -year accumulate survival rates for hMLH1-positive and -negative patients (38.90 and $33.30 \%$, respectively). High CHD1L expression and low hMLH1 expression levels were observed in patients with cholangiocarcinoma, and their abnormal expression patterns were associated with the progression of malignancy and an unfavorable disease prognosis. Therefore, CHD1L and hMLH1 may be potential prognostic biomarkers for cholangiocarcinoma.

\section{Introduction}

Cholangiocarcinoma is the most common malignancy of the bile duct epithelium (1). The global incidence of cholangiocarcinoma has increased from 0.32 to 0.85 per 100,000 individuals within last 30 years, an increase of $165 \%$ (2). Cholangiocarcinoma has become the second primary form of liver cancer after hepatocellular carcinoma, accounting for $10-15 \%$ of total primary liver cancer cases (3). However, the pathogenesis of the majority cholangiocarcinoma cases remains unclear; various high-risk factors have been associated with disease progression, including parasitic infection, primary sclerosing cholangitis, bile duct cysts, intrahepatic bile duct stones, toxins, inflammatory enteropathy, hepatitis B or C virus infection, liver cirrhosis, diabetes, obesity, alcohol consumption, tobacco and genetic polymorphisms (4). Cholangiocarcinoma is characterized by latency in its early stages, which contributes to the challenges of diagnosis, high levels of malignancy and rapid disease progression, leading to an unfavorable prognosis (5). Therefore, the identification of accurate novel molecular biomarkers is of substantial importance for early disease diagnosis, determination of the tumor differentiation stage and the prediction of prognosis.

$\mathrm{CHD} 1 \mathrm{~L}$ is an oncogene that was identified and cloned from the 1q21 chromosome region of human liver carcinoma cells in 1991. It has a full length of 2,980 bp, encoding an $89-\mathrm{kDa}$ protein. CHD1L was observed to exert critical functions in the transcriptional regulation, chromosomal remodeling and 
protein-DNA interaction manipulation (6). Ahel et al (7) reported that CHD1L participated in DNA damage repair, interacting with the DNA damage site through an ATPase domain, dissociating from repaired DNA. The ATPase domain may loosen chromatin via interaction with poly ADP ribose to facilitate DNA repair. However, the overexpression of CHD1L could reduce the degree of chromatin loosening in tumor cells, which may result in the mismatch of DNA bases, potentially initiating tumorigenesis (7). A previous study determined that 58-78\% of primary liver cancer cells expressed CHD1L (8). Tian et al (9) reported that CHD1L overexpression was associated with poorer patient prognosis. CHD1L was also detected in colorectal cancer tissue samples by Ji et al (10): The overexpression of CHD1L was associated with a large tumor size, deep tumor invasion and a high histological. Functional studies revealed that overexpression of CHD1L could promote the transfer of cells from G1- to S-phase and inhibit apoptosis, these results indicating that CHD1L serves notable roles in the pathogenesis of colorectal cancer. Su et al (11), evidenced by quantitative polymerase chain reaction (PCR) and western blot analyses, revealed that CHD1L was highly expressed in gastric cancer, and the overexpression of CHD1L was closely associated with remote metastasis and an unfavorable patient prognosis.

The DNA mismatch repair (MMR) system is activated once DNA damage is detected, so the integrity and stability of DNA can be maintained (12). The loss of function for DNA MMR could occur upon mutation of a DNA MMR system gene, leading to increased rates of DNA base pair mismatch and activator mutations in proto-oncogenes, or the inactivation of tumor suppressor genes, which eventually results in tumor progression (12). hMLH1 is one of the most important members of the DNA MMR system, identified by Bronner et al (13) during their investigation into hereditary non-polyposis colorectal cancer (HNPCC). hMLH1 is located at the 3p21.3-23 chromosomal region and encodes a 756 amino acid-long protein. The hMLH1 gene product was observed to interact with the mismatched gene together with repair enzymes, enhancing DNA repair (13). The downregulation of hMLH1 (including mutation, methylation and loss of heterozygosity) could lead to the loss of DNA MMR function and promote tumor progress (13). Zhang et al (14) reported that 90\% of HNPCC cases were closely associated with mutations to hMLH1. The rate of hMLH1 loss was associated with the invasive depth of colon cancer, as evidenced by Gu et al (15) in their study on 195 patients with colon cancer. The latest research findings indicate that the methylation status of the hMLH1 gene may be of significance for evaluating the risk of recurrence in rectal cancer (16).

The objective of the present study was to investigate CHD1L and hMLH1 expression in patients with cholangiocarcinoma using immunohistological analysis; this was evaluated along with the signs of clinical manifestation and prognosis in order to evaluate their potential prognostic value.

\section{Materials and methods}

Patients and clinicopathological parameters. The present study was approved by the Ethics Committee of The Second Affiliated Hospital of Nanchang University, (Nanchang,
China). The formalin-fixed (Specimens were fixed in 10\% formalin for $10 \mathrm{~min}$ at room temperature), $4-\mu \mathrm{m}$-thick paraffin-embedded cholangiocarcinoma tissues were obtained from 108 patients who were admitted to The Second Affiliated Hospital of Nanchang University (Nanchang, China) between the May 2005 and May 2014 [including 84 male and 24 female patients, with a mean age of 58.26 (range, $40-76$ years)]. Normal bile duct tissues, collected $5 \mathrm{~cm}$ away from the tumor site, were obtained from 60 of the 108 patients. Written informed consent was provided by each participant. The inclusion criteria for patients included a diagnosis of cholangiocarcinoma prior to surgery, confirmed postoperatively, and no preoperative chemotherapy and radiotherapy administered. Patients with concurrent benign bile duct tumors or hepatocellular carcinoma were excluded from the study. Data regarding the clinicopathological parameters were also collected, including age, gender, gallstone history, hepatitis B virus surface antigen (HBsAg) presence, carbohydrate antigen 19-9 (CA19-9) serum level, tumor diameter and Tumor-Node-Metastasis (TNM) stage (Table I). TNM was assessed according to the definitions of the American Joint Committee on Cancer (AJCC), 7th edition (17).

Immunohistochemical analysis. The expression of CHD1L and hMLH1 in cholangiocarcinoma and normal tissues was measured by using PV-9000 Two-Step Immunohistochemical Detection kit (Shanghai Ruiqi Technology Co., Ltd., Shanghai, China).

Rabbit anti-human MLH1 polyclonal antibodies (cat. no. 1167-1-AP; ProteinTech Group, Inc., Chicago, IL, USA; dilution, $1: 100,4^{\circ} \mathrm{C}$ overnight) and rabbit anti-human CHD1L polyclonal antibodies (cat. no. PAB22069; Abnova, Taipei, Taiwan; dilution, $1: 100,4^{\circ} \mathrm{C}$ overnight) were used as primary antibodies, combined with an immunohistochemical MaxVision $^{\mathrm{TM}}$ kit (goat anti-rabbit IgG polymer; cat. no. KIT-5006; dilution, 1:500; Fuzhou Maixin Biotech Co. Ltd., Fuzhou, China), used according to the protocol of the manufacturer, for the detection of MLH1 and CHD1L. Primary antibodies were substituted with PBS for the negative controls and healthy tissues were used for the positive controls. The method of analysis was adapted from that of Plevová et al (18). The immunohistochemical staining was defined according to the stain intensity and number of positive cells from randomly selected five vision fields (x200 magnification) under an inverted fluorescence microscopy (CKX41; Olympus Corporation, Tokyo, Japan).

The number of positive cells were scored as follows: 0 , no positive cells; $1, \leq 25 \%$ of cells positive; $2,26-50 \%$ positive cells; $3,51-75 \%$ positive cells; $4,>75 \%$ positive cells. The staining intensity was scored as follows: 0 , colorless; 1 , light yellow; 2, dark yellow; 4, brown. The final number was the sum of the scores for the proportion of positive cells and the staining intensity: $0-3$, defined as (-); 4-5, defined as (+); 6-7 defined as (++); (+) and (++) were regarded as positive results.

Statistical analysis. SPSS v.17.0 software (SPSS, Inc., Chicago, IL, USA) was used to perform statistical analysis in the current study. The data were analyzed using the $\chi^{2}$ test. The 3 -year overall survival rate was assessed using the Kaplan-Meier 
Table I. Expression of CHD1L and hMLH1 and their association with clinicopathological characteristics.

\begin{tabular}{|c|c|c|c|c|c|c|c|}
\hline \multirow[b]{2}{*}{ Clinical manifestation } & \multirow[b]{2}{*}{ Patients, $n$} & \multicolumn{3}{|c|}{ CHD1L } & \multicolumn{3}{|c|}{ hMLH1 } \\
\hline & & Negative, $\mathrm{n}$ & Positive, $\mathrm{n}$ & P-value & Negative, $\mathrm{n}$ & Positive, $\mathrm{n}$ & P-value \\
\hline \multicolumn{8}{|l|}{$\operatorname{Sex}$} \\
\hline Male & 84 & 6 & 78 & 0.178 & 12 & 72 & $<0.001$ \\
\hline Female & 24 & 0 & 24 & & 12 & 12 & \\
\hline \multicolumn{8}{|l|}{ Age, years } \\
\hline$<60$ & 78 & 6 & 72 & 0.118 & 12 & 66 & 0.006 \\
\hline$\geq 60$ & 30 & 0 & 30 & & 12 & 18 & \\
\hline \multicolumn{8}{|l|}{ Gallstone history } \\
\hline Yes & 48 & 6 & 42 & 0.005 & 6 & 42 & 0.300 \\
\hline No & 60 & 0 & 60 & & 18 & 42 & \\
\hline \multicolumn{8}{|l|}{ HBsAg } \\
\hline Negative & 90 & 6 & 84 & 0.260 & 12 & 78 & $<0.001$ \\
\hline Positive & 18 & 0 & 18 & & 12 & 6 & \\
\hline \multicolumn{8}{|l|}{ CA19-9, U/ml } \\
\hline$<400$ & 30 & 6 & 24 & 0.000 & 12 & 18 & 0.006 \\
\hline$\geq 400$ & 78 & 0 & 78 & & 12 & 66 & \\
\hline \multicolumn{8}{|l|}{ TNM stage } \\
\hline Stage I & 36 & 0 & 36 & 0.000 & 12 & 24 & $<0.001$ \\
\hline Stage II & 24 & 0 & 24 & & 0 & 24 & \\
\hline Stage III & 24 & 6 & 18 & & 12 & 12 & \\
\hline Stage IV & 24 & 0 & 24 & & 0 & 24 & \\
\hline \multicolumn{8}{|l|}{ Tumor diameter, cm } \\
\hline$<4$ & 72 & 6 & 66 & 0.075 & 12 & 60 & 0.050 \\
\hline$\geq 4$ & 36 & 0 & 36 & & 12 & 24 & \\
\hline
\end{tabular}

CHD1L, chromodomain helicase/ATPase DNA binding protein 1; hMLH1, human mutL homolog 1; CA19-9, carbohydrate antigen 19-9; TNM, Tumor-Node-Metastasis.

method, and the inter-group comparisons were performed using the log-rank test. $\mathrm{P}<0.05$ was considered to indicate a statistically significant difference.

\section{Results}

Expression of CHDIL in cholangiocarcinoma. The rate of CHD1L expression was $94.44 \%$ (34/36) in cholangiocarcinoma tissues, compared with only $40.00 \%(8 / 20)$ in non-cancerous tissues, a statistically significant difference $(\mathrm{P}<0.05$; Table II; Fig. 1).

Association between the expression of CHDIL or hMLH1 in cholangiocarcinoma and clinical manifestations. The expression of CHD1L was significantly associated with a history of gallstones, the serum CA19-9 level and TNM staging $(\mathrm{P}<0.05)$. No significant association was determined to be present with gender, age, the presence of HBsAg or the tumor diameter $(\mathrm{P}>0.05)$. hMLH1 expression levels in cholangiocarcinoma $(28 / 36,77.78 \%)$ were significantly lower than those in non-cancerous bile duct tissues $(58 / 60,96.67 \%)$, as determined via the aforementioned immunohistological analysis
(Table III; Fig. 2). The expression of hMLH1 was significantly associated with gender $(\mathrm{P}<0.001)$, age $(\mathrm{P}=0.006)$, serum CA19-9 level $(\mathrm{P}=0.006)$, the presence of HBsAg $(\mathrm{P}<0.001)$, TNM staging and the tumor diameter $(\mathrm{P}<0.05)$, but not with a history of gallstones $(\mathrm{P}>0.05)$.

Survival analysis for the CHDIL-positive and-negative groups, and the hMLH1-positive and-negative groups. A total of 50 patients were randomly selected from the 108 and were followed up for between 1 and 3 years. In total, 26/50 patients were lost during follow-up, and subsequently excluded from the analysis. Of the remaining 24 patients, 18 were CHD1L-positive and 6 were CHD1L-negative patients and 12 were hMLH1-positive and 12 were hMLH1-negative patients. Of these $9 / 24$ patients $(37.5 \%)$ survived to the end of the follow-up period, of whom 4 were CHD1L-positive and 5 were CHD1L-negative, 7 were hMLH1-positive and 2 were hMLH1-negative. The Kaplan-Meier survival curve indicated that the 3-year accumulative survival rate of CHD1L-positive patients was $17.90 \%$, which was significantly lower than for CHD1L-negative patients (83.33\%; P<0.05; Fig. 3). Furthermore, the 3-year overall 
Table II. Comparison of chromodomain helicase/ATPase DNA binding protein 1 expression levels between cholangiocarcinoma patients and healthy controls.

\begin{tabular}{lccccr}
\hline Group & Negative & Positive & Total & Positive rate, \% & P-value \\
\hline Cholangiocarcinoma & 6 & 102 & 108 & 94.44 & $<0.001$ \\
Normal tissue & 36 & 24 & 60 & 40.00 & \\
\hline
\end{tabular}

Table III. Comparison of human mutL homolog 1 expression levels between cholangiocarcinoma patients and healthy controls.

\begin{tabular}{lccccc}
\hline Group & Negative & Positive & Total & Positive rate, $\%$ & P-value \\
\hline Cholangiocarcinoma & 24 & 84 & 108 & 77.78 & $<0.01$ \\
Normal tissue & 2 & 58 & 60 & 96.67 & \\
\hline
\end{tabular}

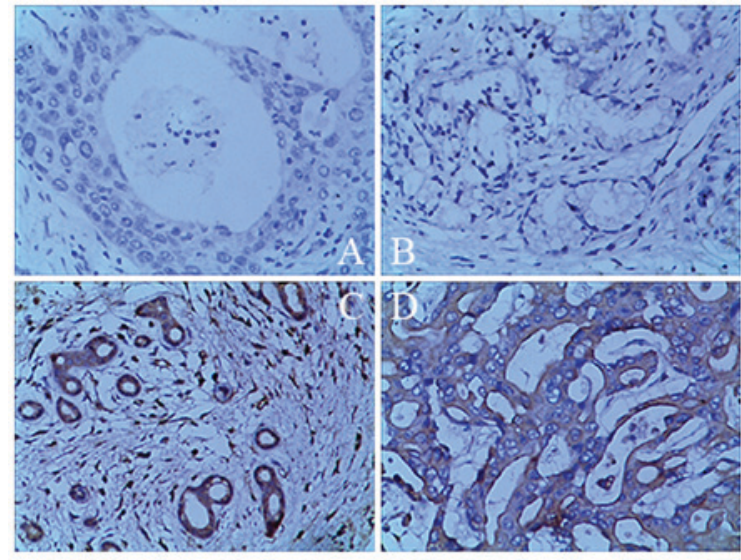

Figure 1. Immunohistochemical staining for CHD1L (brown color). (A) CHD1L-negative in normal bile duct tissue. (B) CHD1L-negative in cholangiocarcinoma tissue. (C) CHD1L-positive in normal bile duct tissue. (D) CHD1L-positive in cholangiocarcinoma tissue. Magnification, $\mathrm{x} 400$. CHD1L, chromodomain helicase/ATPase DNA binding protein 1-like.

survival rate of the hMLH1-positive patients was $38.90 \%$, higher than for the hMLH1-negative patients (33.30\%), although this was not a statistically significant difference ( $\mathrm{P}>0.05$; Fig. 4).

\section{Discussion}

The genesis and development of cholangiocarcinoma are complicated processes with multiple steps and factors involved, including oncogene activation and the loss of tumor suppressor genes (19). Elucidation of the genes essential for cholangiocarcinoma initiation and progression would be of considerable value for disease diagnosis, prognosis and identifying disease progression (1). Recent studies have revealed that the expression levels of CHD1L are high in cases of glioma (20) and lung adenocarcinoma (21), and are associated with tumor diagnosis, advanced clinical stage and prognosis (22). In the current study, the high expression levels of CHD1L in cholangiocarcinoma and the low expression levels in non-cancerous tissues were detected with

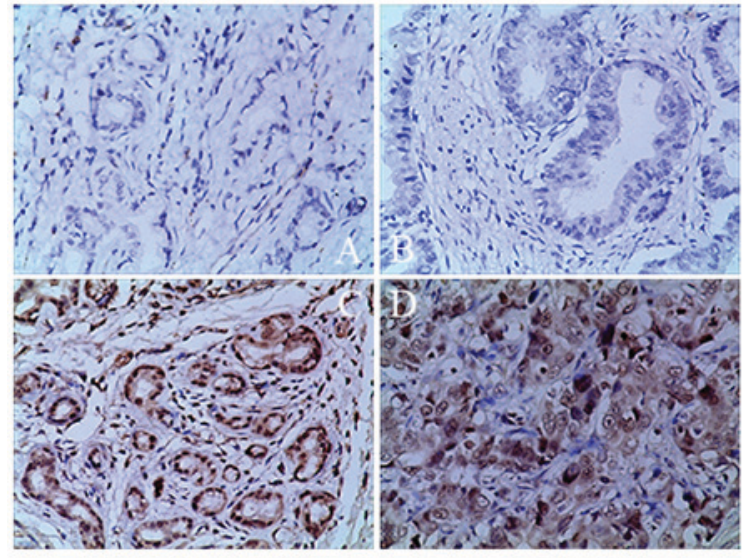

Figure 2. Immunohistochemical staining for hMLH1 (brown color). (A) hMLH1-negative in normal bile duct tissue. (B) hMLH1-negative in cholangiocarcinoma tissue. (C) hMLH1-positive in normal bile duct tissue. (D) hMLH1-positive in cholangiocarcinoma. Magnification, x400. hMLH1, human mutL homolog 1 .

immunohistochemical analysis. Further analysis indicated that high expression of CHD1L was significantly associated with gallstone history, CA19-9 level and TNM stage $(\mathrm{P}<0.05)$, and negatively associated with the overall patient survival rate.

According to a number of studies, hMLH1 was expressed in multiple types of malignancies at relatively low levels (23-26). Giedl et al (23) established that expression of hMLH1 was decreased in early onset bladder cancer, as confirmed by immunohistological analyses. Park et al (24) also obtained similar results using samples of sporadic rectal cancer tissues collected from 318 patients. In addition, decreased expression of hMLH1 was detected in primary non-small cell lung cancer cells using quantitative polymerase chain reaction (25). Through various immunohistochemical methods, Nam et al (26) determined that the low expression levels of hMLH1 in esophageal squamous cell carcinoma cells are associated with patient prognosis.

In the current study, the expression of hMLH1 in cholangiocarcinoma and non-cancerous tissues was assessed 


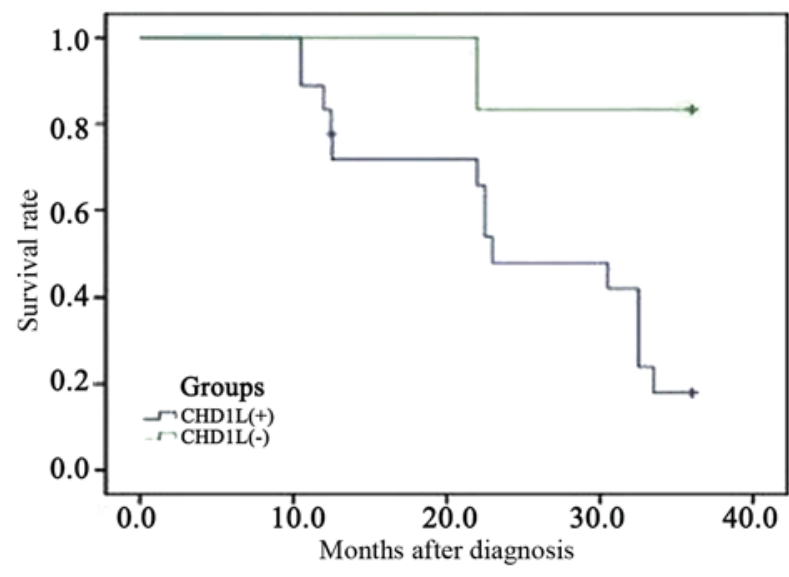

Figure 3. Comparison of the survival rates between chromodomain helicase/ATPase DNA binding protein 1-like-positive and -negative cholangiocarcinoma patients.

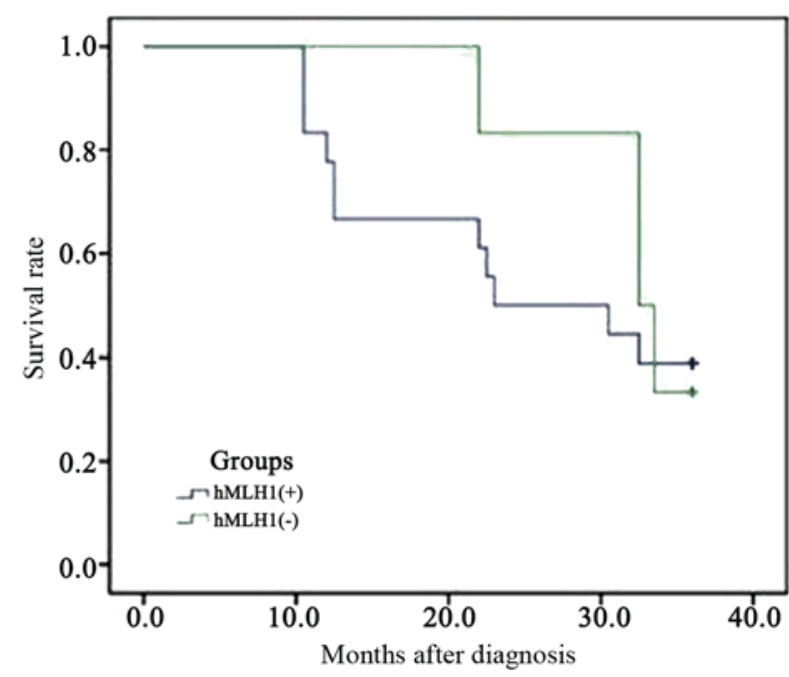

Figure 4. Comparison of the survival rates between human mutL homolog 1-positive and -negative cholangiocarcinoma patients.

immunohistochemically. The expression of hMLH1 was significantly lower in cholangiocarcinoma tissues than in the adjacent normal tissues. Further analysis of expression patterns combined with clinicopathological characteristics including sex, age, gallstone history, HBsAg, CA19-9, TNM stage and tumor diameter, revealed that low expression levels of hMLH1 are significantly associated with age, gender, CA19-9 level, the presence of HBsAg, the TNM stage and tumor diameter. Kaplan-Meier survival curve analysis indicated that patients with lower expression levels of hMLH1 had shorter overall survival periods compared with patients with higher expression levels of hMLH1, although this was not a significant difference. However, the findings of the present study indicated that hMLH1 could be considered as a potential biomarker for prognosis of cholangiocarcinoma.

In conclusion, the results of the present study indicated that high expression levels of CHD1L and low expression levels of hMLH1 are present in cholangiocarcinoma tissues, and that their abnormal expression profile is closely associated with disease development and an unfavorable prognosis. However, the current study was retrospective, and further in vivo and in vitro studies are required in order to investigate the mechanisms by which CHD1L and hMLH1 are involved in the pathological process and affect the tumor invasion and clinical prognosis. Comprehensive research on CDH1L and hMLH1 could contribute to the development of novel advanced methods for the diagnosis and treatment of cholangiocarcinoma.

\section{Acknowledgements}

The authors would like to thank the Second Affiliated Hospital of Nanchang University for assistance with the experiments.

\section{Funding}

This work was supported by the Natural Science Foundation of Jiangxi province, China (grant no. 20151512070090), the Science and Technology Program of Health and Planning Commission in Jiangxi Province (grant no. 20161048) and the Graduate Innovation Fund for Nanchang University (grant no. cx2015203).

\section{Availability of data and materials}

All data generated or analyzed during this study are included in this published article.

\section{Authors' contributions}

$\mathrm{CH}$ was responsible for the experimental design, the funding application and the supervision and management of the project. JH contributed to the execution of experiments, data statistics, manuscript composition and submission. SL participated in performing the experiment in the manuscript mapping, the discussion and data interpretation. All authors have contributed to and approved the final manuscript.

\section{Ethics approval and consent to participate}

The present study was approved by the Ethics Committee of The Second Affiliated Hospital of Nanchang University, (Nanchang, China). Written informed consent was provided by each participant.

\section{Patient consent for publication}

Written informed consent was provided by each participant for publication.

\section{Competing interests}

The authors declare that they have no competing interests.

\section{References}

1. Ghouri YA, Mian I and Blechacz B: Cancer review: Cholangiocarcinoma. J Carcinog 14: 1, 2015.

2. Wadsworth CA, Lim A, Taylor-Robinson SD and Khan SA: The risk factors and diagnosis of cholangiocarcinoma. Hepatol Int 7: 377-393, 2013. 
3. Eckel F, Brunner T and Jelic S; ESMO Guidelines Working Group: Biliary cancer: ESMO clinical practice guidelines for diagnosis, treatment and follow-up. Ann Oncol 22 (Suppl 6): vi40-vi44, 2011

4. Tyson GL and El-Serag HB: Risk factors for cholangiocarcinoma. Hepatology 54: 173-184, 2011.

5. Schweitzer N and Vogel A: Systemic therapy of cholangiocarcinoma: From chemotherapy to targeted therapies. Best Pract Res Clin Gastroenterol 29: 345-353, 2015.

6. Chen L, Chan TH, Yuan YF, Hu L, Huang J, Ma S, Wang J, Dong SS, Tang KH, Xie D, et al: CHD1L promotes hepatocellular carcinoma progression and metastasis in mice and is associated with these processes in human patients. J Clin Invest 120 : $1178-1191,2010$

7. Ahel D, Horejsí Z, Wiechens N, Polo SE, Garcia-Wilson E, Ahel I, Flynn H, Skehel M, West SC, Jackson SP, et al: Poly(ADP-ribose)-dependentregulation ofDNArepairby the chromatin remodeling enzyme ALC1. Science 325: 1240-1243, 2009.

8. He WP, Zhou J, Cai MY, Xiao XS, Liao YJ, Kung HF, Guan XY, Xie D and Yang GF: CHD1L protein is overexpressed in human ovarian carcinomas and is a novel predictive biomarker for patients survival. BMC Cancer 12: 437, 2012.

9. Tian F, Xu F, Zhang ZY, Ge JP, Wei ZF, Xu XF and Cheng W: Expression of CHD1L in bladder cancer and its influence on prognosis and survival. Tumour Biol 34: 3687-3690, 2013.

10. Ji X, Li J, Zhu L, Cai J, Zhang J, Qu Y, Zhang H, Liu B, Zhao R and Zhu Z: CHD1L promotes tumor progression and predicts survival in colorectal carcinoma. J Surg Res 185: 84-91, 2013.

11. Su Z, Zhao J, Xian G, Geng W, Rong Z, Wu Y and Qin C: CHD1L is a novel independent prognostic factor for gastric cancer. Clin Transl Oncol 16: 702-707, 2014.

12. Yan S, Sorrell M and Berman Z: Functional interplay between ATM/ATR-mediated DNA damage response and DNA repair pathways in oxidative stress. Cell Mol Life Sci 71: 3951-3967, 2014.

13. Bronner CE, Baker SM, Morrison PT, Warren G, Smith LG, Lescoe MK, Kane M, Earabino C, Lipford J, Lindblom A, et al: Mutation in the DNA mismatch repair gene homologue hMLH1 is associated with hereditary non-polyposis colon cancer. Nature 368: 258-261, 1994

14. Zhang R, Qin W, Xu GL, Zeng FF and Li CX: A meta-analysis of the prevalence of somatic mutations in the hMLH1 and hMSH2 genes in colorectal cancer. Colorectal Dis 14: e80-e89, 2012.

15. Gu MJ, Bae YK, Kim A, Hong SM, Yu E, Kim J, Jang KT, Chang HK, Jung ES, Bae HI, et al: Expression of hMLH1, hMSH 2 and hMSH6 in small intestinal carcinomas. Hepatogastroenterology 59: 2228-2232, 2012.

16. Kuan JC, Wu CC, Sun CA, Chu CM, Lin FG, Hsu CH, Kan PC, Lin SC, Yang T and Chou YC: DNA methylation combinations in adjacent normal colon tissue predict cancer recurrence: Evidence from a clinical cohort study. PLoS One 10: e0123396, 2015.
17. Kamarajah SK, Burns WR, Frankel TL, Cho CS and Nathan H: Validation of the American Joint Commission on Cancer (AJCC) 8th Edition Staging System for Patients with Pancreatic Adenocarcinoma: A Surveillance, Epidemiology and End Results (SEER) Analysis. Ann Surg Oncol 24: 2023-2030, 2017.

18. Plevová P, Krepelová A, Papezová M, Sedláková E, Curík R, Foretová L, Navrátilová M, Novotný J, Zapletalová J, Palas J, et al: Immunohistochemical detection of the hMLH1 and hMSH2 proteins in hereditary non-polyposis colon cancer and sporadic colon cancer. Neoplasma 51: 275-284, 2004.

19. Gil-García B and Baladrón V: The complex role of $\mathrm{NOTCH}$ receptors and their ligands in the development of hepatoblastoma, cholangiocarcinoma and hepatocellular carcinoma. Biol Cell 108: 29-40, 2016.

20. Sun J, Zhang L, Zhao H, Qiu X, Chen W, Wang D, Ban N, Fan S, Shen C, Xia X, et al: CHD1L regulates cell cycle, apoptosis, and migration in glioma. Cell Mol Neurobiol 36: 565-576, 2016.

21. He LR, Ma NF, Chen JW, Li BK, Guan XY, Liu MZ and Xie D: Overexpression of CHD1L is positively associated with metastasis of lung adenocarcinoma and predicts patients poor survival. Oncotarget 6: 31181-31190, 2015.

22. Wu J, Zong Y, Fei X, Chen X, Huang O, He J, Chen W, Li Y, Shen $\mathrm{K}$ and Zhu L: Presence of CHD1L over-expression is associated with aggressive tumor biology and is a novel prognostic biomarker for patient survival in human breast cancer. PLoS One 9: e98673, 2014.

23. Giedl J, Schneckenpointner R, Filbeck T, Ruemmele P, Hofstaedter F, Burger M, Hartmann A and Stoehr R: Low frequency of HNPCC-associated microsatellite instability and aberrant MMR protein expression in early-onset bladder cancer. Am J Clin Pathol 142: 634-639, 2014.

24. Park JW, Chang HJ, Park S, Kim BC, Kim DY, Baek JY, Kim SY, Oh JH, Choi HS, Park SC and Jeong SY: Absence of hMLH1 or hMSH2 expression as a stage-dependent prognostic factor in sporadic colorectal cancers. Ann Surg Oncol 17: 2839-2846, 2010.

25. VageliD,DaniilZ,Dahabreh J,KaragianniE,VamvakopoulouDN, Ioannou MG, Scarpinato K, Vamvakopoulos NC, Gourgoulianis KI and Koukoulis GK: Phenotypic mismatch repair $\mathrm{hMSH} 2$ and $\mathrm{hMLH} 1$ gene expression profiles in primary non-small cell lung carcinomas. Lung Cancer 64: 282-288, 2009.

26. Nam TK, Lee JH, Cho SH, Chung IJ, Ahn SJ, Song JY, Yoon MS, Chung WK and Nah BS: Low hMLH1 expression prior to definitive chemoradiotherapy predicts poor prognosis in esophageal squamous cell carcinoma. Cancer Lett 260: 109-117, 2008.

This work is licensed under a Creative Commons Attribution-NonCommercial-NoDerivatives 4.0 International (CC BY-NC-ND 4.0) License. 